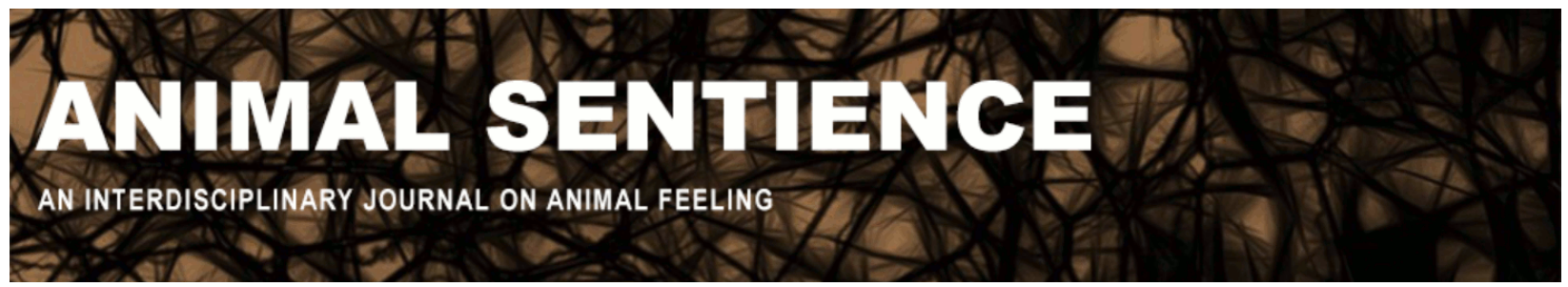

Kramer, Leo Bernd (2017) What does it feel like to be an electroreceptive fish?. Animal Sentience 8(3)

DOI: $10.51291 / 2377-7478.1188$

Date of submission: 2017-01-18

Date of acceptance: 2017-02-01

(c)

This article has appeared in the journal Animal

Sentience, a peer-reviewed journal on animal

cognition and feeling. It has been made open access,

free for all, by WellBeing International and deposited

in the WBI Studies Repository. For more information,

please contact

wbisr-info@wellbeingintl.org.

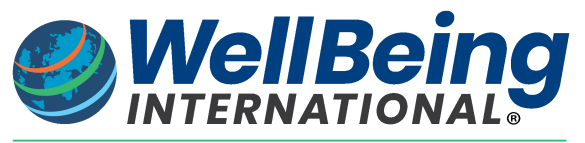

SOLUTIONS FOR PEOPLE, ANIMALS AND ENVIRONMENT 


\title{
What does it feel like to be an electroreceptive fish?
}

\author{
Commentary on Balcombe on Fish Knows
}

\author{
Leo Bernd Kramer \\ Zoological Institute \\ University of Regensburg, Germany
}

\begin{abstract}
The weakly electric knifefish Eigenmannia emits an electric organ discharge (EOD) of constant frequency and sinusoidal waveform that varies with sex and age. Eigenmannia discriminates among these except when stimulated at the same frequency as its own EOD frequency. In that case, it needs to perform a Jamming Avoidance Response (frequency shift) which results in a beating mixed signal. By a sophisticated analysis of the amplitude and phase modulations of the beat signal, Eigenmannia derives the frequency difference, its sign, and the waveform of the stimulus, hence the signaller's identity. The human ear is not capable of an equivalent waveform analysis of acoustic stimuli.
\end{abstract}

Keywords: animal communication, signal analysis, fish cognition

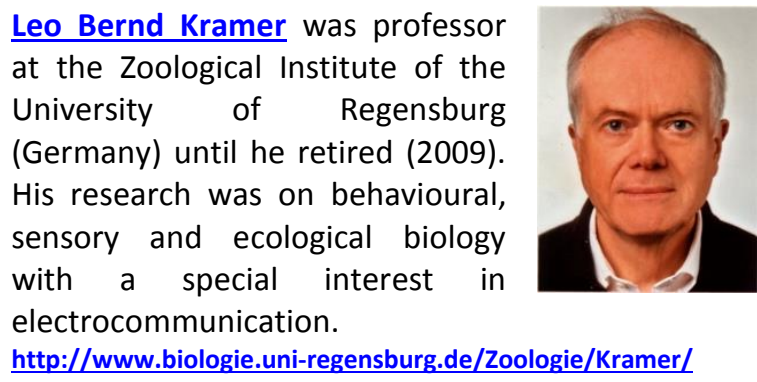

I have studied the sensory and behavioral biology of fish for 45 years and continue to admire their beauty, intelligence and sophistication. Yet the great Herbert Melville is quite outspoken about fish in Mardi: Of all nature's animated kingdoms, fish are the most unchristian, inhospitable, heartless, and cold-blooded of creatures. Could he be right? For all we know, fish have neither religion nor empathy, even though we cannot be sure about the latter. In his book What a Fish Knows Jonathan Balcombe $(2016 a, b)$ discusses the growing evidence for sentience in "our underwater cousins." He addresses questions such as: Can they feel pain? Do they possess consciousness and awareness? Balcombe argues that cognition in fish is unjustifiably and grossly underrated compared to cognition in other vertebrates and that we should stop regarding fish as the unfeeling sources of protein for our dinner table, or free throw-away material in angling contests.

This article gives an example supporting Balcombe's plea from research on the glass knifefish Eigenmannia virescens, which lives in fresh water in tropical South America. I have observed large groups of Eigenmannia hiding during the day under the platforms of house boats anchored on the shore of the Rio Negro at Manaus, or in reed beds near islands or the banks of the mighty Solimoes (Amazon), only moving out into the open water at night. These delicate fish and their relatives are electroreceptive and electrogenic, as we know only since around 1960, and for me they are one of the top wonders "in nature's animated kingdoms" (Melville).To listen to their harmonious choruses of "electrical voices" (made audible by a 
headset) in a rowing boat on the Solimoes at night made me wish to study the sensory behaviour and ecology of these mysterious creatures and their new sensory capacity (new to us humans). One of the questions I addressed as a young postdoc in the laboratory of Tomas Szabo at the C.N.R.S. research institution near Paris was "do these fish communicate electrically with one another"? At that time "communication in fish" was considered an anthropomorphic question by most scientists (not Tomas), not amenable to science in the strictest sense.

The electric sense in fish means the presence of cutaneous electroreceptor organs of which the sensory cells are connected to specialized hindbrain structures by afferent nerves. Electroreceptive fish detect field strengths down to the 1 microvolt/cm to 5 nanovolt/cm range (for freshwater and marine environments, respectively). (A 1.5 Volt Mignon battery and a lot of wire are sufficient to set up an electric field strength of $300 \mu \mathrm{V} / \mathrm{cm}$ in a swimming pool of Olympic dimensions, of $50 \mathrm{~m}$ length.) The electric sense must have arisen in the distant ancestors of vertebrates; thus, all the major living classes of fish and even some amphibia are considered electroreceptive by common descent. Some descendant groups have lost common electroreception, among them the speciose teleosts or bony fishes, frogs, reptiles, birds and mammals (reviewed in Kramer 1996, 2009a-c).

The South American knifefishes (Gymnotiformes) and the African snoutfishes (Mormyridae) are electroreceptive bony fishes (teleosts) and live in freshwater bodies of South America and Africa, respectively. Independently, their ancestors have re-evolved electroreception and combined it with an electric effector organ that generates electric fields the strength of which remains just below detectability by non-electroreceptive organisms, such as most other teleosts. (The strongly electric eel is an exception among the otherwise weakly electric knifefishes.) Phylogenetically and embryologically, the electric organ is derived from modified muscle tissue in most species.

This electric sender-receiver system is of dual use: (1) the nocturnal fish test objects or obstacles in the near field (centimeters) with their self-generated electric field for the impedance properties of objects. This system is called Active Electrolocation (AE) and is reminiscent of the echolocation system of bats, but unlike in bats, there are no echoes involved in electroreception. (Knifefish and snoutfish also detect foreign sources of weak electricity, such as those emanating from other live organisms close by: this passive electrolocation is also present in the many non-electrogenic, electroreceptive fishes such as sturgeons or sharks.) (2) Knifefish and snoutfish communicate electrically with other group members in a much wider perimeter than AE (several meters). Both fish clades lead a nocturnal life, and thus are able to avoid the diurnal, visually orientated predators, such as piranhas and tigerfish, who abound in tropical fresh waters.

The knifefish Eigenmannia virescens emits a constant-frequency electric organ discharge (EOD) of around 250-600 Hertz that is one of Nature's most stable signal sources. Displayed on an oscilloscope, one notes the sinusoidal signal waveform (sinusoidal means "periodical, and similar in waveform to a sine wave"; Figure 1). When Eigenmannia is stimulated with a sine wave of a frequency similar to its own it tends to shift its frequency away from the stimulus (Watanabe \& Takeda 1963). This response was later called a "Jamming Avoidance Response (JAR)," a term borrowed from radio frequency engineers (Bullock et al. 1972). The response was described as reflexive (automatic, or "unconscious," and never habituating). It was thought to enhance the discrimination of a fish's own signal from those of nearby, "jamming" conspecifics, thereby affording better active electrolocation of objects. The neuroethology of the JAR was studied in a long series of 
detailed papers mainly on brain physiology, and the JAR was said to represent "the first vertebrate behavior the neuroethology of which was completely understood, from receptors to effectors" (Heiligenberg 1991). However, early on some instances of deviating ("not typical") JAR responses and purported "free will" of Eigenmannia had also been reported (Scheich 1977). The subsequent demonstration of strong JAR habituation in a sterile experimental lab situation, and clear differences in JAR behavior by study subjects of different sex or age, did not fit the picture. For example, big Eigenmannia males tended to be electrically unresponsive, and preferred to physically attack another real or simulated fish of similar frequency. Females and juveniles tended to increase their frequency, even when the stimulus was somewhat higher than their own (Kramer 1985, 1987). Frequency increases were observed in subdominant fish and are thought to represent submissive signals in Eigenmannia ethology (Hagedorn \& Heiligenberg 1985).
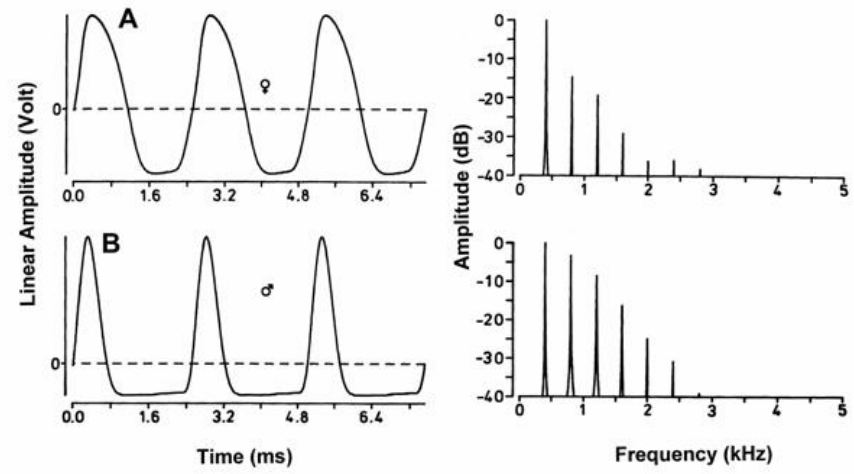

\begin{abstract}
Figure 1. Waveforms (A,B; left) of electric organ discharges in Eigenmannia, with their associated Fourier amplitude spectra (to their right). The ordinates of the left-hand diagrams are arbitrary linear amplitudes $(\mathrm{V})$, those of the right-hand diagrams are amplitudes expressed as $\mathrm{dB}$ attenuation relative to the strongest spectral component of each waveform (which is the first harmonic or fundamental in both cases). (A) Female; (B) male. Time in milliseconds, frequency in kilohertz. Note the almost sinusoidal waveform of the female electric organ discharge (EOD), the higher harmonics of which are of much weaker amplitude than in the male EOD, whose waveform deviates markedly from a sinusoid (from Kramer 1999).
\end{abstract}

Laboratory experiments with Eigenmannia showed that performing a JAR did not notably enhance its active electrolocation performance when stimulated with a "jamming" signal of EOD frequency. Even when frequency-clamped to the EOD, the fish's $A E$ performance was nearly unimpaired, irrespective of JAR (Heiligenberg 1977; cf. Kramer 1996, pp. 84-91). By contrast, being allowed to perform a JAR to an unclamped stimulus that was initially of EOD frequency did make all the difference to trained, food-rewarded fish in identifying the stimulus waveform, that is, in electro-communication. When the positive (rewarded) stimulus $\mathrm{S}+$ was presented, trained fish first performed a big JAR and then swam to the feeder to get the reward (Figure 2A). The negative stimulus $S$ - of identical spectral composition and intensity but different waveform was unrewarded, and, after training, fish remained in their hiding pot (Figure $2 \mathrm{E}$ ). When the stimuli S+ and S- were frequency-clamped to the fish's own frequency, following it dynamically, the fish were unable to discriminate the positive from the negative signal and chance responses were observed (Kramer 1999). 

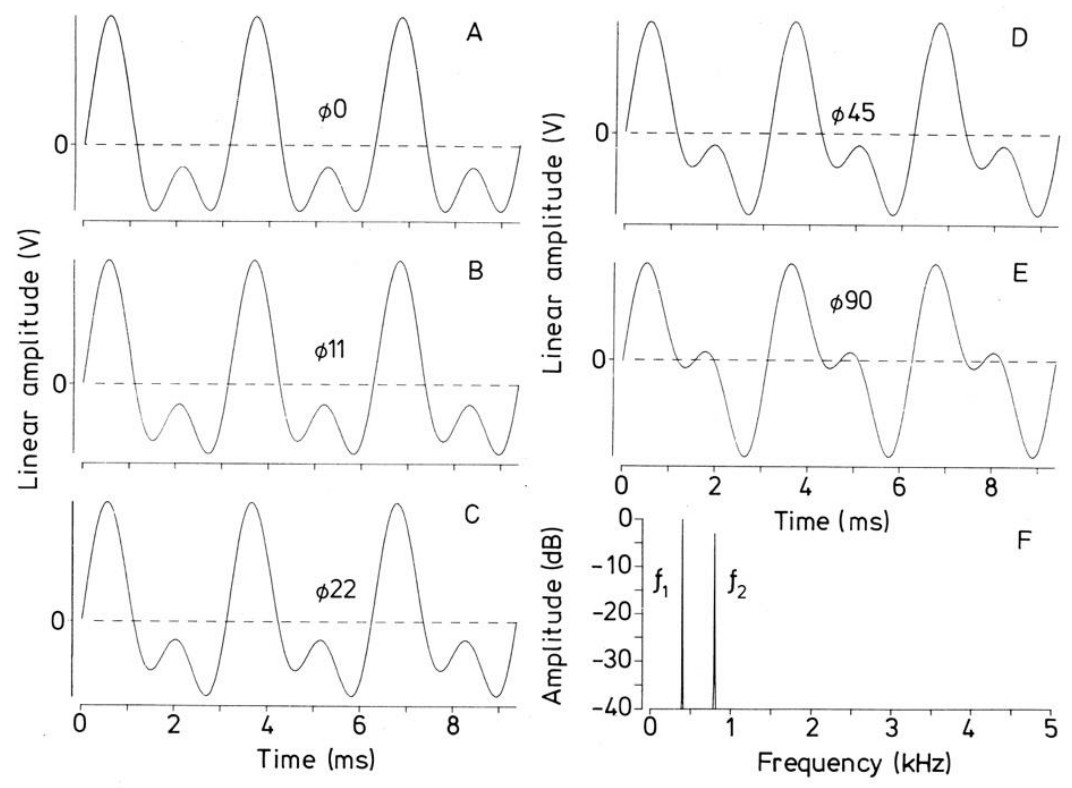

Figure 2. Stimulus waveforms $(A-E)$, all of identical amplitude spectrum $(F)$, as used in training experiments with Eigenmannia. Waveforms are composed of two harmonically related sine waves (a strong fundamental, $f 1$, that is superimposed by its harmonic, $f 2$, of twice that frequency and with an amplitude of $-3 \mathrm{~dB}$ ). Time in milliseconds, frequency in kilohertz. A-E differ only in the phase $(\phi)$ difference between $f 1$ and $f 2$. Here $\phi 0$ designates the temporal coincidence of the peaks of the two sine waves (arbitrarily called " $0^{\circ}$ phase difference") and $\phi 90$ designates a delay of a quarter of a cycle $\left(90^{\circ}\right)$ (from Kramer 1999).

In order to discriminate the positive from the negative stimulus, the fish needs a minimum frequency difference between its own EOD and the stimulus, that is, it needs to perform a JAR, which is only possible with an unclamped stimulus. The addition of the two signals in the water leads to a mixed signal periodically beating at the difference frequency, the amplitude and phase modulation of which is analyzed by the fish. The fish not only estimates the frequency difference from the beating signal, including its sign, but also the waveform of the stimulus, which is not directly available because of the mixing of the signals (Figure 3). The fish turns the disadvantage into an advantage: by analyzing the beat signal for the waveform of the stimulus (rather than the stimulus itself), the speed requirement is greatly relaxed. At $400 \mathrm{~Hz}$ the duration of one period (or cycle) of the sinusoidal Eigenmannia EOD is 2.5 milliseconds; at a stimulus frequency of (say) plus or minus $4 \mathrm{~Hz}$ of the EOD frequency, the beat period is 250 milliseconds. A sensory mechanism has been proposed for waveform discrimination based on the so-called tuberous electroreceptor organs of two types, the amplitude-sensitive $P$ receptors and the phase-sensitive $T$ receptors. The waveform of the stimulus is reconstructed by a plot of the difference between T-receptor response times from both sides of the body over one beat period (time; reviewed in Kramer 1996). A fast neural circuit has been discovered which compares left and right (as well as tail and head) T receptor information (reviewed in Carr 1990). 


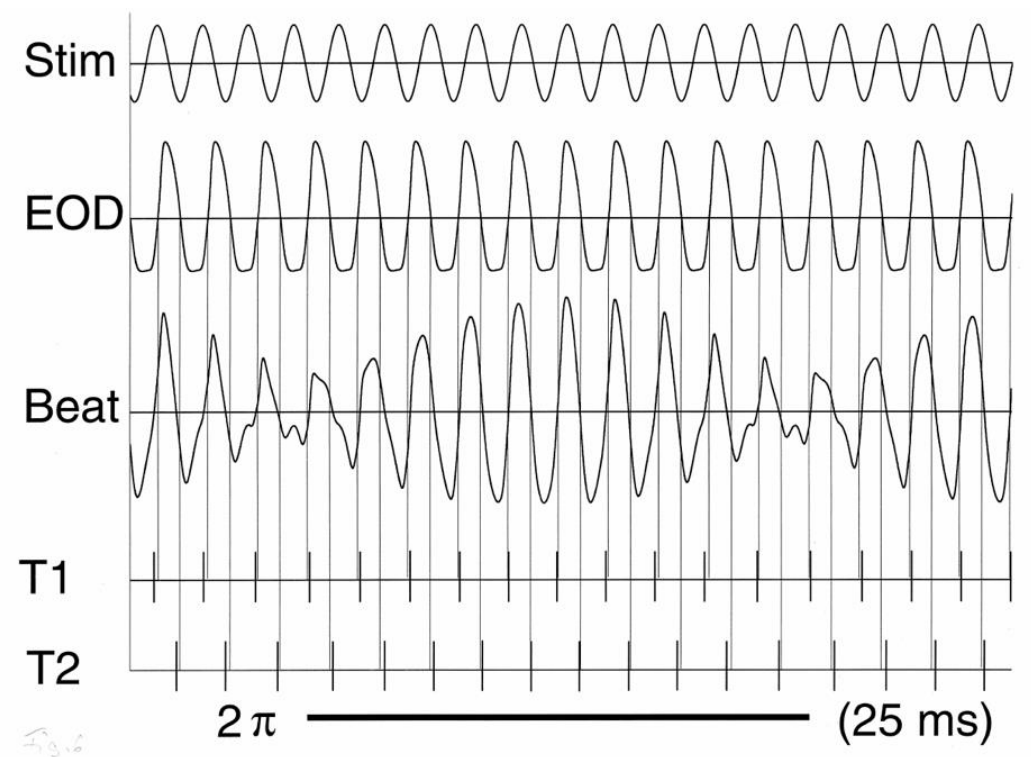

Figure 3. Stim A sine wave stimulus (of $440 \mathrm{~Hz}$ and $60 \%$ amplitude) and an Eigenmannia EOD (electric organ discharge of a female, of $400 \mathrm{~Hz}$ and $100 \%$ amplitude) are superimposed (Beat, third line). T1 and $T 2$ show sensory afferences from $T$ electroreceptors, $T 1$ responding to positive-going zerocrossings of the superposition signal, $T 2$ to negative-going ones (on opposite body sides). Note temporal disparities of action potentials with regard to zero-crossings in the original EOD (thin vertical reference lines). When beat amplitude rises, zero-crossings lag; when the amplitude declines, they are leading relative to the EOD. At a $\Delta \mathrm{f}$ or difference frequency of $40 \mathrm{~Hz}$ (chosen for clarity) the duration of one beat cycle, $2 \pi$, is $25 \mathrm{~ms}$, constituting 10 EOD cycles; no JAR is evoked. A more realistic $\Delta \mathrm{f}$ of $4 \mathrm{~Hz}$, however, usually evokes a strong JAR, and the duration of one beat cycle is $250 \mathrm{~ms}$, constituting 100 EOD cycles (from Kramer 2001).

Eigenmannia individuals differ in EOD waveform: juveniles have an almost sinusoidal waveform, adult females' EODs deviate more clearly from a sine wave, and males' EODs deviate the most. The deviations can be expressed by the $\mathrm{P} / \mathrm{N}$ ratio, that is, the duration of the positive-going half-wave of the EOD over the negative half-wave (the voltage integral over time is zero in Eigenmannia's EOD). As the negative half-wave is longer than the positive in Eigenmannia's EOD, the $\mathrm{P} / \mathrm{N}$ ratio is $<1$. A perfect sine wave is $\mathrm{P} / \mathrm{N}=1$, because positive and negative half-waves are mirror images of one another. Any sinusoidal wave signal of $\mathrm{P} / \mathrm{N}<1$, or not a perfect sinusoid, carries higher harmonics to the fundamental frequency. According to French mathematician Joseph Fourier (1768-1830), in a periodic signal (that deviates from a sine wave), harmonics are integer multiples of the fundamental frequency, that is, the series of harmonics in a typical Eigenmannia EOD would go like this: $400 \mathrm{~Hz}$ (the fundamental), $800 \mathrm{~Hz}, 1,200 \mathrm{~Hz}$ (and so on). In all Eigenmannia individuals, the EOD fundamental frequency is the strongest, with rapidly decreasing amplitudes the higher the harmonic. The content in higher harmonics (total harmonic distortion) is strongest in adult males, weakest in juveniles. The harmonics (or overtones) in an Eigenmannia EOD are characterized by their amplitudes relative to the fundamental and their phase or temporal relationships. An Eigenmannia male EOD, when made audible, sounds bright, like a violin tone (rich in overtones); a juvenile's EOD sounds duller, like a flute (poor in overtones).

The human ear is incapable of discriminating sound signals of identical harmonic content but different in waveform (different because of the shifted phase relationships among harmonics); by contrast, for an electric signal, Eigenmannia can do so. Compare the 
live microphone output of a sound signal with its tape recording on an oscilloscope screen with two beams: you will see totally different waveforms in spite of acoustic identity as far as our ears are concerned. This is because the direct-recording tape technique introduces frequency-dependent phase shifts that change signal waveform without affecting the spectral amplitude composition. What would be a hi-fi tape recording that was good enough for us humans would not pass for Eigenmannia.

Eigenmannia is a social fish; it is well equipped to discriminate the EODs of a whole bunch of conspecifics around it, using (1) "differences in harmonic content," that is, timbre, which we humans also detect in a sound signal. Eigenmannia is, in addition, sensitive to (2) the "phase relationship between harmonics," that is, the signal waveform (not detected by the human ear in a sound signal). It is as if Eigenmannia had its own built-in oscilloscope in its (for a fish) extra-large brain, a feat humans had to solve technically by the invention of the oscilloscope. There is a physical reason we humans cannot have an acoustic equivalent of Eigenmannia's sensory super hi-fi capacity for electrical signals: the speed of sound in air (or water) varies with frequency; the speed of electrical signals does not (i.e., for all practical purposes, it is independent both of frequency and the medium). Therefore, both the waveform of a sound signal and its spectral amplitude composition (if harmonics are present) change with the receiver's distance from the sender; for an electrical signal, they do not change.

It is not known how or when the ancestors of Eigenmannia "discovered" that the electric communication channel offered reliable waveform fidelity, and how they capitalized on it by evolving far more than a hundred species, all with characteristically differing EOD waveforms. The Gymnotiformes is turning out to be a large and ever growing order of fish as scientists continue to discover new species in their studies on natural communities in South America. Most (perhaps all) species have species-specific and sometimes sex-specific EOD waveforms for their local communities that are detected and, most likely, significant in these fishes' lives. There is still much to learn about the secretive, nocturnal lives of these marvelous creatures the complexity of which we are only beginning to fathom. Is it possible to deny Eigenmannia sentience and awareness when it keeps track of the individually varying EOD frequencies and waveforms of its conspecifics by sophisticated signal analysis? while also showing long-term memory, personality and awareness of its own spatial position and social rank relative to group members? I think this would be a big mistake.

\section{References}

Balcombe, J. (2016a). What a fish knows: The inner lives of our underwater cousins. New York: Farrar, Straus and Giroux.

Balcombe, J. (2016b). In praise of fishes: Précis of What a fish knows (Balcombe 2016). Animal Sentience, 8(1).

Bullock, T.H., Hamstra, R.H. \& Scheich, H. (1972). The jamming avoidance response of high frequency electric fish. I. General features. Journal of Comparative Physiology A 77:1-22.

Carr, C.E. (1990). Neuroethology of electric fish. Principles of coding and processing sensory information. BioScience 40:259-267.

Hagedorn, M. \& Heiligenberg, W. (1985). Court and spark: Electric signals in the courtship and mating of gymnotoid fish. Animal Behaviour 33:254-265. 
Heiligenberg, W. (1977). Principles of electrolocation and jamming avoidance in electric fish. Berlin, Heidelberg, New York: Springer-Verlag.

Heiligenberg, W. (1991). Neural nets in electric fish. Cambridge, MA: MIT Press.

Kramer, B. (1985). Jamming avoidance in the electric fish Eigenmannia: harmonic analysis of sexually dimorphic waves. The Journal of Experimental Biology 119:41-69.

Kramer, B. (1987). The sexually dimorphic jamming avoidance response in the electric fish Eigenmannia (Teleostei, Gymnotiformes). The Journal of Experimental Biology 130:39-62.

Kramer, B. (1996). Electroreception and communication in fishes. Stuttgart, Jena, Lübeck, Ulm: Gustav Fischer Verlag.

Kramer, B. (1999). Waveform discrimination, phase sensitivity and jamming avoidance in a wave-type electric fish. The Journal of Experimental Biology 202:1387-1398.

Kramer, B. (2001). Signal analysis in the communication of a weakly electric fish, Eigenmannia virescens (Gymnotiformes). In W. Backhaus (Ed.), Neuronal coding of perceptual systems (pp. 314-333). New Jersey, London, Singapore, Hong Kong: World Scientific.

Kramer, B. (2009a). Electric communication and electrolocation. In M.D. Binder, N. Hirokawa, \& U. Windhorst (Eds.), Encyclopedia of neuroscience (pp. 1038-1045). Berlin, Heidelberg: Springer-Verlag.

Kramer, B. (2009b). Electric fish. In M.D. Binder, N. Hirokawa, \& U. Windhorst (Eds.), Encyclopedia of neuroscience (pp. 1045-1050). Berlin, Heidelberg: Springer Verlag.

Kramer, B. (2009c). Electric organ discharge. In M.D. Binder, N. Hirokawa, \& U. Windhorst (Eds.), Encyclopedia of neuroscience (pp. 1050-1056). Berlin, Heidelberg: Springer Verlag.

Scheich, H. (1977). Neural basis of communication in the high frequency electric fish, Eigenmannia virescens (jamming avoidance response). I. Open loop experiments and the time domain concept of signal analysis. Journal of Comparative Physiology $A$ 113:181-206.

Watanabe, A. \& Takeda, K. (1963). The change of discharge frequency by a.c. stimulus in a weak electric fish. The Journal of Experimental Biology 40:57-66. 